\title{
Dinâmica do crescimento de árvores dominantes em povoamentos clonais de Tectona grandis Linn. F.
}

Anna Carolina Simão Pinheiro ${ }^{1 *}$, João Paulo Sardo Madi ${ }^{1}$, Mariana Peres de Lima Chaves e Carvalho ${ }^{1}$, Adriano Ribeiro de Mendonça ${ }^{2}$, Valdir Carlos Lima de Andrade ${ }^{3}$, Diogo Guido Streck Vendruscolo ${ }^{1}$, Marcos Felipe Nicoletti ${ }^{4}$, Samuel de Pádua Chaves e Carvalho ${ }^{1}$

${ }^{1}$ Universidade Federal de Mato Grosso, Rua Fernando Corrêa da Costa, 2367, Boa Esperança, Cuiabá - MT, 78068-600

${ }^{2}$ Universidade Federal do Espirito Santo, Alto Universitário, s/n ${ }^{\circ}$, Guararema, Alegre - ES, 29500-000

${ }^{3}$ Universidade Federal do Tocantins, Rua Badejós, Lote 7, Chácaras 69/72 Zona Rural, Gurupi-TO, 77402-970

${ }^{4}$ Universidade do Estado de Santa Catarina, Avenida Luiz de Camões, 2090, Conta Dinheiro, Lages - SC, 88520-000

*Autor para correspondência: annacspinheiro@gmail.com

Recebido: Setembro 2019 / Aceito: Novembro 2019 / Publicado: Dezembro 2019

\section{Resumo}

A classificação de sítios florestais é umas etapas fundamentais no manejo de plantios florestais, e se dá principalmente pela média das alturas das árvores dominantes. O objetivo deste trabalho foi avaliar a dinâmica das árvores dominantes na classificação da capacidade produtiva de sítios florestais e verificar se a estrutura sugerida por Schumacher é estatisticamente indicada para descrever o crescimento em altura dominante de árvores clonais de teca. Para este fim foram abordadas duas formas distintas de classificação de árvore dominante. A primeira que fixa estas árvores na primeira medição da parcela de inventário florestal, sendo, portanto, uma única classificação durante toda a rotação do povoamento. A segunda que classifica uma árvore dominante em cada medição da parcela, e posterior cálculo da altura médias das árvores dominantes. Nesta pesquisa os dados foram originados do monitoramento contínuo de 121 unidades amostrais de campo, com idade variando de 1,1 a 8,1 anos, de plantios comerciais de Tectona grandis Linn. F, localizados na região Centro-Sul do estado de Mato Grosso. Para fins de comparação das duas abordagens do estudo, foram utilizadas as estatísticas de coeficiente de determinação $\left(\mathrm{R}^{2}\right)$ e o erro padrão em escala percentual. As estatísticas dos modelos foram complementadas pela análise da estabilidade das unidades amostrais na rotação do povoamento, e também pela identidade das curvas. As estatísticas de ambos os métodos de classificação foram muito próximas, com $\mathrm{R}^{2}$ de $0,9950 \mathrm{e}$ 0,9953 , e erro padrão residual de 22,56 e $21,48 \%$. Quanto à estabilidade, o modelo de Schumacher, para ambos os métodos, classificou como estável mais de $90 \%$ das parcelas utilizadas no estudo. E sobre a identidade das curvas, pôde-se inferir que ambas as curvas de sítios foram similares na classificação da capacidade produtiva. Portanto, foi possível concluir que não existe diferença estatística entre as curvas de sítios quando consideradas as diferentes classificações de árvores dominantes, o que comprova a hipótese de que árvores dominantes se mantém dominantes durante toda a rotação florestal.

Palavras-chave: Capacidade produtiva, Identidade de Modelos, Dinâmica Florestal, Teca.

\footnotetext{
Abstract

The classification of forest sites is a fundamental stage in management of forest plantations, and this is calculate by median of dominant height of trees. The objective of this work was to evaluate the dynamics of dominant trees in the classification of productive capacity of forest stands and evaluate if the Schumacher's model is statistically indicated to describe the growth of dominant height of clonal teak trees. For this purpose two distinct forms of dominant tree classification were discussed. A first fixes the dominant trees in the first measurement of the forest inventory, being,
}

therefore, a single classification throughout the forest rotation. A second one that classifies a dominant tree in each measurement of the each plots, and later calculation of the average height of the dominant trees. The data used in this study came from of 121 plots, with age 1,1 to 8,1 years from commercial teak plantations, located in the south-central region of the state of Mato Grosso. For purposes of comparison of the procedures covered in this study, the stability of the plots in the classification as well as the identity of the curves were evaluated. Similar values of coefficient of determination $\left(R_{2}\right)$ and standard error percentage $\left(S_{y x} \%\right)$ were observed for both methods. The models evaluated were efficient to describe the growth in dominant height for the evaluated stands. The model identity test indicated that the dominant height growth was similar by the two approaches brought in this study, confirming the hypothesis that dominant trees remain dominant throughout the forest rotation.

Kevwords: Forest productivity, Identity model, Forest Dynamics, Teak.

\section{Introdução}

A Teca é uma espécie com alto valor agregado, fácil de ser cultivada e, e sua madeira apresenta diversos usos, o que justifica o estabelecimento de plantios no Brasil e no mundo (Flórez, 2012). Com o crescimento do cultivo da teca, ocorre também o interesse em desenvolver estudos específicos para a espécie em questão, como é o caso de estudos de crescimento de árvores individuais e dos povoamentos.

A árvore é influenciada pelos fatores genéticos integrados com os fatores edafoclimáticos e a competição com outros indivíduos. A interação de todos esses fatores sobre a planta expressa a qualidade do sítio, representada pelo índice de sítio, cujo método mais praticado e difundido na determinação de capacidade de produção de povoamentos florestais (Batista et al., 2009).

Segundo Scolforo (1997), existem vários métodos para classificar a capacidade produtiva de um local. No entanto, a classificação da produtividade por meio de métodos diretos onde a classificação produtiva é medida por meio do crescimento da floresta, utilizando dados de altura dominante, é considerada a forma mais prática e usual, já que essa variável é pouco influenciada pelos desbastes por baixo e não ser afetada pela densidade do povoamento.

Tendo em vista a importância da classificação da capacidade de produção de um sítio florestal, este estudo tem como objetivo avaliar o efeito na classificação final da capacidade produtiva pela seleção das árvores dominantes de acordo com dois métodos sugeridos, sendo o primeiro seguindo o critério de Assmann e o segundo mantendo as árvores dominantes da primeira medição nas consecutivas, além de verificar a hipótese de que árvores dominantes permanecem dominantes durante o ciclo florestal, assim 
como, avaliar se a estrutura sugerida pelo modelo desenvolvido por Schumacher é estatisticamente indicada para descrever o crescimento em altura dominante de árvores clonais de teca.

\section{Material e métodos}

$\mathrm{O}$ estudo foi realizado em plantios clonais de Tectona grandis Linn. F., localizados na região Centro-Sul de Mato Grosso, microrregião do Alto Pantanal, com altitude média de 118 m. O clima da região, segundo Koppen (1931)é do tipo Awi (Savanas tropicais com verão úmido e inverno seco). As médias de temperatura anual são $32^{\circ} \mathrm{C}$ para máxima e $21^{\circ} \mathrm{C}$ para mínima. Também possui uma nítida estação seca, apresentando déficit hídrico entre abril e outubro.

A fim de avaliar a dinâmica das árvores dominantes na classificação de sítios florestais, foram sugeridos dois métodos de classificação:

MÉTODO 1: Consiste na classificação de árvores dominantes segundo o principio de Assmann (1970), em que altura dominante é a média das alturas das 100 árvores com maior diâmetro por hectare. Seguindo esse princípio a classificação é feita a partir de inventários florestais sucessionais realizados anualmente onde são selecionadas as árvores com maior diâmetro de forma proporcional a área da unidade amostral e, posteriormente, é obtida a média destas alturas. Esse procedimento é realizado a cada novo inventário forestal a fim de se obter a altura dominante da unidade amostral na idade da medição.

MÉTODO 2: Esta sugestão de classificação também segue o principio de Assmann (1970), porém com modificações a partir da segunda medição. A altura dominante da unidade amostral resultante da primeira medição é obtida de maneira identica a do método 1 . Nas medições subsequentes, mantem asárvores classificadas como dominantes na primeira medição para o cálculo da altura dominante média das parcelas nas idades subsequentes.

Os dados que compuseram o estudo foram originados da medição de 120 unidades amostrais, com idade variando entre 1,1 e 8,1 anos. As medições foram realizadas em 19 talhões que somados totalizam 592,58 hectares.

Para a classificação dos sítios florestais, inicialmente foi ajustado o modelo de Schumacher (1939) (Eq. 1).

$\operatorname{Ln}\left(\mathrm{HD}_{\mathrm{ij}}\right)=\beta_{0}+\beta_{1}\left(\frac{1}{\mathrm{I}_{\mathrm{ij}}}\right)+\varepsilon_{\mathrm{ij}}$

em que: $H D_{i j}=$ altura dominante média da i-ésima parcela na j-ésima medição; $I_{i j}=$ idade da i-ésima parcela na j-ésima medição; $\beta_{\mathrm{i}}=$ parâmetros a serem estimados; $\varepsilon_{i j}=$ erro da $\mathrm{i}-$ ésima parcela na j-ésima medição.

Como próximo passo, utilizou-se o método da equação da diferença (Clutter et al. 1983) (Eq. 2) para a projeção das parcelas e posterior classificação dos sítios.

$\operatorname{Ln}\left(\operatorname{Hd}_{2}\right)=\operatorname{Ln}\left(\operatorname{Hd}_{1}\right)+\beta_{1}\left[\left(\mathrm{I}_{2}^{-1}\right)-\left(\mathrm{I}_{1}^{-1}\right)\right]$ em que: $H d_{2}=$ Altura dominante na medição i+1; $H d_{1}=$ Altura dominante na i-ésima medição; $I_{1}^{-1}=$ inverso da idade na i-ésima medição; $I_{2}^{-1}=$ inverso da idade na medição i+1; $\beta_{\mathrm{i}}=$ parâmetros específico do sítio estimado por regressão

De acordo com Campos e Leite (2009), as expressões para construção das curvas e estimativas do sítio para cada parcela, pelo método da equação das diferenças, podem ser obtidas por meio de pares consecutivos de idade e altura dominante, determinando as diferenças sucessivas entre $\mathrm{LnHd}_{2}-\mathrm{LnHd}_{1}$ e $_{2}^{-1}-\mathrm{I}_{1}^{-1}$, em que $1 \mathrm{e} 2$ se referem à idade atual e imediatamente posterior, respectivamente. Após isso, ajusta-se a regressão linear simples $\mathrm{Y}=\mathrm{f}(\mathrm{X})$, onde $\mathrm{Y}=\mathrm{Ln}\left(\mathrm{Hd}_{2}\right)-\left(\mathrm{LnHd}_{1}\right)$ e $\mathrm{X}=\mathrm{I}_{2}^{-1}-\mathrm{I}_{1}^{-1}$, resultando na Equação 2.

Igualando-se $I_{2}$ à idade índice, tem-se que $H d_{2}$ é igual ao índice de sítio. Esta foi a estrutura utilizada (Eq.3) para a construção das curvas de sítio pelo método da equação das diferenças.

$\operatorname{Ln}(\mathrm{IS})=\operatorname{Ln}(\mathrm{Hd})+\beta_{1}\left[\left(\mathrm{I}_{2}^{-1}\right)-\left(\mathrm{I}_{1}^{-1}\right)\right](3)$

em que: $H d=$ Altura dominante na idade $I_{1} ; I S=$ Índice de sítio; $\beta_{1}=$ parâmetro específico do sítio estimado por regressão; $I_{1}^{-1}=$ Idade de medição; $I_{2}^{-1}=$ Idade de projeção ou índice.

As equações foram avaliadas por meio do Coeficiente de determinação (Eq. 4), Erro padrão da estimativa (Eq. 5 e 6) e análise gráfica dos erros em escala percentual (Eq. 7).

$\mathrm{R}^{2}=\left(\frac{\mathrm{SQ}_{\mathrm{ras}}}{\mathrm{SQ}_{\mathrm{wo}}}\right)$

$\mathrm{S}_{\mathrm{yx}}=\sqrt{\frac{\sum_{\mathrm{i}=1}^{\mathrm{n}}\left(\mathrm{y}_{\mathrm{i}}-\hat{\mathrm{y}}_{\mathrm{i}}\right)}{\mathrm{n}-\mathrm{p}}}$

$\mathrm{S}_{\mathrm{yx}} \%=\frac{\mathrm{S}_{\mathrm{yx}}}{\overline{\mathrm{y}}} .100$

$\mathrm{E}_{\%}=\left(\frac{\mathrm{y}_{\mathrm{i}}-\hat{\mathrm{y}}_{\mathrm{i}}}{\mathrm{y}_{\mathrm{i}}}\right) \cdot 100$

Em que: $y i=$ altura dominante observada; $y \hat{i}=$ altura dominante estimada; $\bar{y}=$ média aritmética das alturas dominantes $(\mathrm{m}) ; \mathrm{n}=$ número de observações; $\mathrm{p}=$ número de parâmetros do modelo; $\mathrm{SQ}_{\mathrm{res}}=$ soma de quadrados do

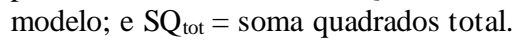

De maneira complementar, foi realizada a avaliação da estabilidade de projeção das parcelas unidades amostrais para os métodos propostos, ou seja, se as unidades amostrais tiveram a sua classificação alterada de um ano para outro ou se manteve na mesma classificação do ano anterior. Uma unidade amostral foi considerada estável quando, nas distintas medições e classificações, esta não tenha flutuado em mais de 1 classe de sítio. As demais situações foram consideradas instáveis. Foi também utilizado o teste de Teste de Identidade(Tabela 1) de modelos a fim de testar a hipótese de igualdade das curvas geradas pelos dois métodos propostos (Regazzi, 1993).

Tabela 1. Estrutura geral do teste de identidade de modelos

\begin{tabular}{llll}
\hline FV & GL & SQ & F \\
\hline Método 1 (M1) & $\mathrm{n}_{1}$ & SQM.1 & \\
\hline Método 2 (M2) & $\mathrm{n}_{2}$ & SQM.2 & \\
\hline Diferença & $\mathrm{n}_{1}-\mathrm{n}_{2}$ & SQD & QMD/QMR
\end{tabular}

em que: $\mathrm{FV}=$ fonte de variação; $\mathrm{GL}=$ grau de liberdade; SQ = soma dos quadrados; $\mathrm{F}=$ teste $\mathrm{F} ; \mathrm{N}=$ número de observações; SQD = soma de quadrados da diferença; $\mathrm{n}=$ número de parâmetros do modelo; M.1 = método $1 ; \mathrm{M} .2=$ 
método SQM.2=SQT- $\sum(y-\hat{y} M 2)^{2}$ e SQD=SQM.1-SQM.2

Portanto, quando o valor da estatística $\mathrm{F}$ calculada for maior ou igual a $\mathrm{F}_{5 \%}\left(\mathrm{n}_{1}, \mathrm{n}_{2}\right)$, seu valor tabelar, indica que não é possível utilizar uma única equação para gerar as curvas de classificação de sítios florestais.

\section{Resultados e discussão}

Os resultados dos ajustes do modelo de Schumacher (1939) estão representados na Tabela 2.

Tabela 2. Análise estatística dos modelos de Schumacher ajustado para os diferentes métodos

\begin{tabular}{lccc}
\hline Método & $\mathbf{S y x}(\mathbf{m})$ & $\mathbf{S y x} \%$ & $\boldsymbol{R}_{\mathbf{2}}$ \\
\hline Método 1 & 1,7490 & 22,56 & 0,9950 \\
\hline Método 2 & 1,6336 & 21,48 & 0,9953 \\
\hline
\end{tabular}

Os resultados da Tabela 2 demonstram que o modelo de Schumacher é adequado para representar o crescimento em altura dominante para os diferentes métodos propostos com erros de estimativas inferiores a 2 metros e grau de explicação acima de $99 \%$, reforçando o que diz o estudo de Dias et al. (2005), no qual o valor do coeficiente de determinação também foi superior a $90 \%$. Porém, ao comparar os valores percentuais do erro padrão da estimativa com os de outros trabalhos, como os de Couto et al. (1989) e Dias et al., (2005), que apresentaram valores do erro padrão percentual abaixo de $10 \%$, resultados superiores em relação aos dois métodos testados.

$\mathrm{Na}$ Figura 1, faz-se a análise gráfica dos resíduos do método 1, e 2, respectivamente. Pode-se demonstrar que, para os valores de altura até $10 \mathrm{~m}$, o modelo tende a superestimar, para os métodos 1 e 2 respectivamente. Para a unidade amostral com valores de altura dominante maior que estes, os dois métodos tendem a subestimar a altura dominante média.

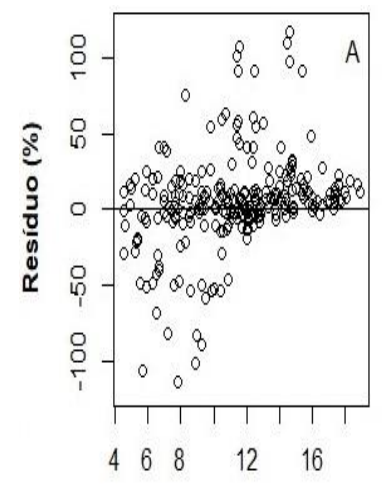

Altura dominante $(\mathrm{m})$

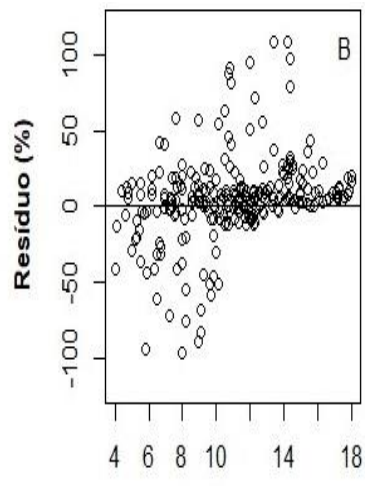

Altura dominante ( $\mathrm{m})$
Figura 1. Gráfico de resíduos percentuais para as equações ajustadas.

Os resultados da Tabela 3 demonstram que o método 1 totalizou 92\% de unidades amostrais estáveis e $8 \%$ de instabilidade. Já o método 2 apresentou $90 \%$ de estabilidade e $10 \%$ de instabilidade.

Os resultados demonstram que ambos os métodos são estáveis, porém o método 2 , se comparado ao método 1 , apesar de apresentar percentual de instabilidade maior que do método 1, pode ser considerado o mais estável já que não possuiu nenhuma parcela com flutuação entre quatro classes distintas no crescimento em altura dominante.

Tabela 3. Análise da estabilidade na projeção para os dois métodos propostos

\begin{tabular}{lcllll}
\hline & \multicolumn{2}{l}{ Total Parcelas } & \multicolumn{2}{l}{ Estabilidade } \\
\hline $\begin{array}{l}\text { Mudança } \\
\text { classe }\end{array}$ & de & $\begin{array}{l}\text { Métod } \\
\text { o 1 }\end{array}$ & $\begin{array}{l}\text { Métod } \\
\text { o 2 }\end{array}$ & $\begin{array}{l}\text { Métod } \\
\text { o 1 }\end{array}$ & $\begin{array}{l}\text { Métod } \\
\text { o 2 }\end{array}$ \\
\hline $\begin{array}{l}\text { Variou } \\
\text { classe }\end{array}$ & uma & 53 & 54 & $44 \%$ & $45 \%$ \\
\hline $\begin{array}{l}\text { Variou } \\
\text { classes }\end{array}$ & duas & 58 & 54 & $48 \%$ & $45 \%$ \\
\hline $\begin{array}{l}\text { Variou } \\
\text { classes }\end{array}$ & três & 8 & 12 & $7 \%$ & $10 \%$ \\
\hline $\begin{array}{l}\text { Variou } \\
\text { classes }\end{array}$ & quatro & 1 & 0 & $1 \%$ & $0 \%$ \\
\hline Total & & & & & \\
\hline
\end{tabular}

Os resultados de estabilidade foram complementados pelo teste de identidade de modelos (Tabela 4). Nota-se que o F calculado $(1,0985)$ é menor que o $F$ tabelado $(1,1824)$, sendo, portanto, não significativo, o que implica que as curvas são semelhantes, e que uma única equação pode ser utilizada para classificar sítios de povoamentos de Teca. A Figura 2 corrobora com os resultados da Tabela 4 em que é possível observar a proximidade das curvas para os diferentes métodos propostos. As linhas contínuas representam as curvas de índice de sítio do método 1 , e as linhas pontilhadas representam as curvas de índice de sítio do método 2 , em que é notável a semelhança entre as curvas de sítio.

Tabela 4. Análise de variância do teste de identidade de modelos

\begin{tabular}{llll}
\hline FV & GL & SQ & $\begin{array}{l}\text { F } \\
\text { tabelado }\end{array}$ \\
\hline Método 1 & 366 & 8,4469 & \\
\hline Método 2 & 362 & 9,5454 & \\
\hline Diferença & 4 & 1,0985 & 1,1824 \\
\hline
\end{tabular}

Com base nas análises estatísticas de estabilidade e do teste de identidade de modelos, verifica-se que os dois métodos de classificação de árvores dominantes propostos geram curvas de índice de sítio semelhantes, sendo possível utilizar apenas uma equação para gerar curvas para os dois métodos propostos. Esses resultados reforçam a hipótese de que as árvores dominantes permanecem dominantes durante a rotação florestal.

Com base nos resultados, sabe-se que independente da escolha do método, as curvas obtidas serão semelhantes e, demonstram que a escolha do método não afetará a classificação final da capacidade de produção. Contudo, recomenda-se o método 2 devido à sua operacionalidade em campo, visto que as medições serão realizadas sempre nas mesmas árvores dispensando a medição de $100 \%$ das unidades amostrais para então selecionar as árvores de maior diâmetro para posterior medição de sua altura. 


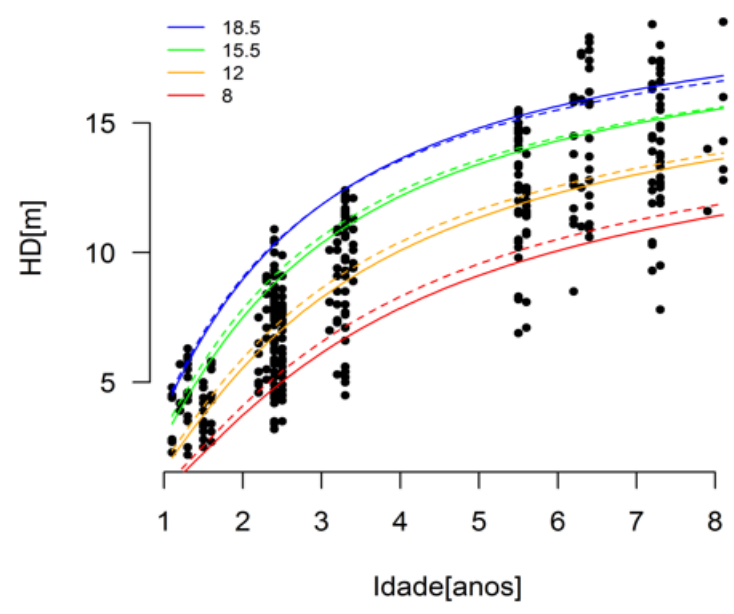

Figura 2. Curvas de índice de sítio referentes aos dois métodos propostos.

\section{Conclusões}

A classificação final da capacidade produtiva pela seleção das árvores dominantes a partir dos dois métodos sugeridos, sendo o primeiro seguindo o critério de Assmann e o segundo mantendo as árvores dominantes da primeira medição nas consecutivas, verificou - se que as árvores dominantes se mantêm dominantes durante toda a rotação florestal, por meio do teste de identidade das curvas de sítios geradas, a partir dos dois métodos testados. Conclui-se ainda que a função de Schumacher é estatisticamente indicada para descrever o crescimento em altura dominante de árvores clonais de teca.

\section{Referências}

Assmann E (1970) The principles of forest yield study. Oxford: Pergamon Press, 506 p.

Batista JLF, Couto HTZ, Silva Filho DF(2014) Quantificação de recursos florestais: árvores, arvoredos e florestas. São Paulo: Oficina de textos, 379p.

Campos CC, Leite HG (2009) Mensuração florestal: perguntas e respostas. Viçosa: UFV, 470p

Clutter JL, Fortson JC, Pienaar LV, Brister GH, Bailey RL (1983) Timber management: A quantitative approach. New York: John Wiley \& Sons, 333p.

Couto HTZ, Bastos NLM, Lacerda JS(1989) Comparação de dois modelos de prognose de madeira de Eucalyptus para alto fuste e talhadia. IPEF, 41/42: 27-35.

Dias, NA; Leite, HG; Nogueira, GS; Rodrigues, FL. Avaliação de métodos de ajuste de curvas de índice de local em povoamentos de eucalipto desbastado. Revista Árvore, v. 29: 741-747, 2005.

Flórez JB (2012) Caracterização tecnológica da madeira jovem de teca (Tectona grandis L.F.). Disertação, (Mestrado em ciência e tecnologia da madeira) $\neg$ Universidade Federeal de Lavras.85 p.

Köppen W (1931) Grundriss der Klimakunde: Outline of climate science. Berlin: Walter de Gruyter, 1931. 388p.

Regazzi AJ (1993) Teste para verificar a identidade de modelos de regressão e a igualdade de alguns parâmetros num modelo polinomial ortogonal. Revista Ceres, v.40 (228):176-195.
Schumacher FXA (1939) New growth curve and its application to timber yield studies. Journal Forestry,37:819-820.

Scolforo JRS (1997) Biometria florestal: métodos para classificação de sítios florestais. Lavras: UFLA / FAEPE,151p. 